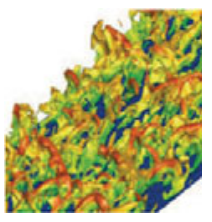

\title{
FOREWORD: Turbulence Colloquium Marseille 2011
}

\author{
Fundamental Problems of Turbulence, 50 years after the Marseille 1961 Conference, \\ Centre International de Rencontres Mathématiques (CIRM), \\ Marseille, 28-30 September 2011 organised by \\ Marie Farge ${ }^{\mathrm{a}}$, H. Keith Moffatt ${ }^{\mathrm{b}}$ and Kai Schneider ${ }^{\mathrm{c}}$ \\ ${ }^{a}$ Ecole Normale Supérieure, Paris, France; ${ }^{b}$ Trinity College, University of Cambridge, Cambridge, \\ United Kingdom; ${ }^{c}$ Aix-Marseille Université, Marseille, France
}

\section{Objective and scientific interest}

Turbulence remains one of the oldest and most challenging research problems in both pure and applied science; recognition of the phenomenon can be traced back to Leonardo da Vinci who introduced the word 'Turbolenza'. Turbulence characterises the flow of a fluid (gas, liquid or plasma) dominated by nonlinear interactions that make its description and the prediction of its evolution exceptionally difficult. Turbulence has been studied for several centuries by mathematicians as well as by physicists and engineers. It is still an open problem since no satisfactory theory is yet available, from either mathematical or physical viewpoint. Moreover, the turbulent regime may not be as universal as commonly supposed.

In 1961, Alexandre Favre organised one of the earliest international colloquia dedicated to turbulence on the occasion of the inauguration of the 'Institut de Mécanique Statistique de la Turbulence (IMST)' that he founded in Marseille. The impact of this colloquium was crucial and continues to play an important role 50 years later. It was attended by outstanding luminaries of the subject: Kolmogorov, Yaglom, von Karman, G.I. Taylor, Liepmann, Laufer, Corrsin, Batchelor, Kovasznay, Kraichnan and many others. Key problems were identified and presented during review lectures given by several invited speakers, followed by extended open discussions [1]. This colloquium led to the development of research areas that are still very much alive to this day.

\section{Short description of the meeting}

The Turbulence Colloquium Marseille 2011 (TCM2011) 'Fundamental problems of turbulence' was organised in order to mark the 50th anniversary of this seminal meeting. It therefore followed the same pattern and adopted a similar long-term perspective. Its goal was to assess the achievements of the last 50 years of turbulence research and to identify future challenges that still remain. There were seven sessions; each devoted to a generic type of turbulent flow, together with three special sessions: a recollection of 'The Turbulence

[1] 'Mécanique de la Turbulence' Proceedings of the International Colloquium held in Marseille, August 28th-September 2nd 1961, Colloques Internationaux du CNRS, 108, 1962. 
Colloquium Marseille 1961 (TCM1961), a presentation of mathematical ideas that arise through the study of turbulence and a final session to discuss new ideas and perspectives.

Just as for TCM1961, nine speakers were invited to present review lectures describing the state-of-the-art in turbulence research; each session began with one of these review lectures, followed by posters and short oral presentations, and concluded with a one-hour open discussion led by the session presidents and recorded by a scientific secretary. More than 80 invited participants from 14 countries attended the colloquium.

TCM2011 was chaired by four honourary presidents: Michel Coantic, Edward Spiegel, Tomomasa Tatsumi and Bryan Taylor. The review lecturers, presidents and scientific secretaries of the various sessions were as follows:

\section{Homogeneous turbulence and flow structure}

Review lecturer: H. Keith Moffatt

Presidents: Toshiyuki Gotoh and Fazle Hussain

Scientific secretaries: Wouter Bos and Michael Wilczek

\section{Shear and wake flow turbulence}

Review lecturers: Garry Brown and Anatol Roshko

Presidents: Robert Antonia and Charles Williamson

Scientific secretaries: Dmitry Kolomenskiy and Paulo Luzzatto-Fegiz

Pipe and channel flow turbulence

Review lecturer: John Kim

Presidents: Bruno Eckhardt and Zhen-Su She

Scientific secretaries: Xi Chen and Paulo Luzzatto-Fegiz

Boundary layer turbulence

Review lecturer: James Wallace

Presidents: Javier Jimenez and Beverley McKeon

Scientific secretaries: Sedat Tardu and Michael Wilczek

Historical session: TCM1961

Review lecturer: Michael Eckert

Presidents: Fabien Anselmet, Patrick Bontoux and Jean-Paul Dussauge

Scientific secretaries: Philip Schäfer and Antoine Venaille

\section{Turbulent stirring and mixing}

Review lecturer: Katepalli Sreenivasan

Presidents: Rahul Pandit and Norbert Peters

Scientific secretaries: Samriddhi Ray and Philip Schäfer

Mathematical session: mathematics for turbulence

Review lecturer: Claude Bardos

Presidents: Charles Doering and Edriss Titi

Scientific secretaries: Dmitry Kolomenskiy and Romain Nguyen van yen

\section{Geophysical turbulence}

Review lecturer: Roddam Narasimha

Presidents: Herman Clercx and Joel Sommeria

Scientific secretaries: Antoine Venaille and Samriddhi Ray

Magneto-hydro-dynamic turbulence

Review lecturers: William Matthaeus and David Montgomery

Presidents: Bérengère Dubrulle and Shigeo Kida

Scientific secretaries: Wouter Bos and Nobumitsu Yokoi

Final session: new ideas and perspectives

Presidents: Eberhard Bodenschatz, Charles Meneveau and Parviz Moin

Scientific secretaries: Lionel Larchevêque and Romain Nguyen van yen

The review lectures and the posters, together with papers, ideas and videos, can be downloaded from the colloquium's website: $h t t p: / / w w w . t u r b u l e n c e . e n s . f r$

The proceedings of TCM1961 [1] have been digitalised, and can also be found on the above address. 


\section{A guided tour through the special issue}

Here is a brief outline of contributions made during TCM2011 and published in this special issue of Journal of Turbulence.

There are eight review papers:

- H. Keith Moffatt reviews how our present understanding of homogeneous turbulence developed over the last 50 years, discussing the theory Kolmogorov presented at TCM1961, intermittency, the finite-time singularity problem, the skewness factor paradox, Kraichnan's direct interaction approximation, helicity and the turbulent dynamo, two-dimensional turbulence, rapid distortion theory, and problems related to passive and active scalar transport.

- Garry Brown and Anatol Roshko describe how turbulent shear layers and wakes are studied, with a particular emphasis on the experimental observation of large-scale vortical structures (coherent structures), and why they seem to play an essential role in understanding the turbulent regime.

- John Kim gives an overview of the progress made since TCM1961 in understanding turbulent flows in pipes and channels, and addresses the unresolved issues that require further research.

- James Wallace presents a detailed survey of experimental and theoretical achievements made since TCM1961 in the study of turbulent boundary layers.

- Michael Eckert shows how turbulence research developed up to TCM1961, detailing the circumstances and the environment in which new concepts emerged.

- Claude Bardos and Edriss Titi review the main theorems related to the Euler, NavierStokes and Prandtl equations that are relevant for studying turbulent flows.

- Roddam Narasimha covers recent developments in our understanding of turbulent atmospheric flows encountered in tropical clouds and in the low-wind convective regime of the atmospheric boundary layer.

- William Matthaeus, David Montgomery, Minping Wan and Sergia Servidio review the magnetohydrodynamics of several turbulent regimes encountered in plasma flows, in particular, for the electron fluid plasma that is closely related to two-dimensional hydrodynamics, and for the solar wind where three-dimensional effects become important.

There are also seven research papers that have been submitted to Journal of Turbulence by participants of TCM2011. These do not cover all topics presented at the colloquium, but focus mainly on the advances in the statistical theory of homogeneous isotropic turbulence, self-consistent closure, strongly anisotropic turbulence, turbulent combustion modelling and Lagrangian dynamics of turbulence.

Bonne lecture!

Marie Farge, H. Keith Moffatt and Kai Schneider

(Paris, Cambridge and Marseille)

The organisers of TCM2011 thankfully acknowledge the financial support from the following organisations:

- Centre International de Rencontres Mathématiques (CIRM).

- Université de la Méditerranée, Marseille.

- Total Company. 
- Institut de Recherche sur la Fusion par confinement Magnétique (IRFM).

- Commissariat à l'Énergie Atomique et aux Énergies Alternatives (CEA).

- Center for Turbulence Research (CTR), Stanford University and NASA-Ames.

- Office of Naval Research Global.

- Groupement de Recherche Européen CNRS, Mécanique des Fluides Numériques.

- Institut National des Sciences de l'Univers (INSU), Centre National de la Recherche Scientifique (CNRS).

- Institut National des Sciences de l'Ingénierie et des Systèmes (INSIS), Centre National de la Recherche Scientifique (CNRS).

- Ecole Centrale de Marseille (ECM).

- European Research Community on Flow, Turbulence And Combustion (ERCOFTAC).

- Association Française de Mécanique (AFM). 